\begin{tabular}{|c|c|}
\hline Title & Learning an accurate entity resolution model from crowdsourced labels \\
\hline Author(s) & Wang, Jingjing; Oyama, Satoshi; Kurihara, Masahito; Kashima, Hisashi \\
\hline Citation & $\begin{array}{l}\text { ICUIMC } 2014 \text { : January 9-11, Siem Reap, Cambodia ; proceedings, ISBN: 978-1-4503-2644-5, 1-8 } \\
\text { https://doi.org/10.1145/2557977.2558060 }\end{array}$ \\
\hline Issue Date & 2014-01-09 \\
\hline Doc URL & http:/hdl.handle.net/2115/65191 \\
\hline Rights & $\begin{array}{l}\text { (2014 ACM. This is the author' sversion of the work. It is posted here by permission of ACM for your personal use. } \\
\text { Not for redistribution. The definitive version was published in PUBLICA TION, ICUIMC '14 Proceedings of the 8th } \\
\text { International Conference on Ubiquitous Information Management and Communication, } \\
\text { http://doi.acm.org/10.1145/2557977.2558060 }\end{array}$ \\
\hline Type & proceedings (author version) \\
\hline File Information & IMCOM2014-WANG.pdf \\
\hline
\end{tabular}

Instructions for use 


\section{Learning an Accurate Entity Resolution Model from Crowdsourced Labels}

\author{
Jingjing Wang \\ Graduate School of \\ Information Science and \\ Technology \\ Hokkaido University \\ Sapporo 060-0814, Japan \\ jingjing.wang@live.cn
}

\author{
Satoshi Oyama \\ Graduate School of \\ Information Science and \\ Technology \\ Hokkaido University \\ Sapporo 060-0814, Japan \\ oyama@ist.hokudai.ac.jp \\ Hisashi Kashima \\ Department of Mathematical \\ Informatics \\ The University of Tokyo \\ Tokyo, Japan / JST PRESTO \\ kashima@mist.i.u- \\ tokyo.ac.jp
}

\author{
Masahito Kurihara \\ Graduate School of \\ Information Science and \\ Technology \\ Hokkaido University \\ Sapporo 060-0814, Japan \\ kurihara@ist.hokudai.ac.jp
}




\section{Planet Explore - Abby Watkins}

www.planetexplore.com/partner_view.php?p=81

Since she began climbing in 1988, Abby has excelled at just about every kind of climbing imaginable. In 1996, she and fellow Australian Vera Wong set the ...

\section{Abby Watkins Illustrations Trendland: Fashion Blog \& Trend}

\section{trendland.com/abby-watkins-illustrations/}

2010.6.12 - Just about to enter her 3rd year of Textile Design for a Fashion degree at Manchester Metropolitan University 21-year-old Abby Watkins, ...

Figure 1: Entity Resolution Problem.

bels from individual worker labels is to use simple majority voting (MV) $[17 ; 18 ; 25]$, which can often achieve relatively good results depending on the accuracy of the workers involved. In MV, the label receiving the most votes is selected as the final aggregated label.

There are a number of papers on designing fundamental algorithms for crowdsourcing $[9 ; 14 ; 15]$ and a number of papers on developing systems for entity resolution in Web search result [13; $1 ; 2 ; 21 ; 12]$, but only a few on crowdsourcing entity resolution [22; 8]. The method proposed by Gomes et al. [9] uses a novel model of human clustering rather than supervised machine learning to aggregate worker annotations, but it cannot be used to predict categories of unknown data. Wang et al. [22] reported a hybrid humanmachine workflow for dealing with crowdsourcing entity resolution: all the data are processed by machines first, and only the most likely matching data are verified manually.

We have compared two supervised machine learning methods for resolving entities: locality preserving projections (LPP) [10] using consensus labels obtained by majority voting and a combination of multiple Laplacians (multi-Lap) using crowd-sourced data directly. Since the feature vectors obtained from Web pages are high dimensional and very sparse, a dimensionality reduction approach is used in the learning process of both methods to project high-dimensional data objects to a low-dimensional latent feature space, and the data objects are identified on the basis of the distance between them. That is, the closer two data objects are to each other in the latent space, the greater the likelihood that they represent the same real world entity.

The LPP method was designed to derive an optimal entity model from actual crowdsourced data without a gold standard. Since individual crowd workers often exhibit highly variable labeling accuracy, we asked the workers to label each HIT so that we could derive a single consensus label by majority voting. If an individual worker labels a pair of Web pages more than once, the label used is the one that has the most votes among the workers. The final consensus label is the one that has the most votes among the workers.

Since majority voting treats each worker's vote equally, we use the multi-Lap method to directly make use of the variable-quality labels generated by crowd workers. To derive an accurate entity model, we added a weighting measure to the learning process: higher weights are assigned to the more accurate workers. Without such weighting, each worker's vote is treated equally. Two weighting measures are used to represent crowd worker credibility. One is based on the assumption the majority vote gives the ground truth label. Thus, we value the weight of each worker as the degree of similarity between the labels provided by the worker and the labels provided by the other workers.

However, under some circumstances, the majority opinion is incorrect. For example, if most of the crowd workers give an incorrect label, the majority opinion must be wrong. Especially with crowdsourcing, the probability is high that the majority of the workers for a particular task are unskilled. We thus use the mutual dependency principles proposed by Dai et al. [5] as our second weighting measure to evaluate crowd worker credibility. These principles include positive and negative mutual dependencies. For our problem, a positive mutual dependency means that a worker is more credible if he gives agreeing edges to pairs of Web pages referring to the same real world entity and vice versa. A negative mutual dependency means that a worker is less credible if he gives disagreeing edges to pairs of Web pages referring to the same real world entity and vice versa.

We experimentally evaluated the label quality of actual crowdsourced data by comparing the labels provided by individual workers and the consensus labels with the ground truth labels. We computed consensus labels by weighting each worker's vote equally (simple MV). Then we compared the prediction accuracy of the conventional LPP method using consensus labels with that of our proposed multi-Lap method with and without weighting.

\section{SUPERVISED MACHINE LEARNING US- ING CROWD-GENERATED LABELS}

Since the feature vectors obtained from Web pages are high dimensional and very sparse, we need to use a dimensionality reduction approach in order to project high-dimensional data objects to a low-dimensional latent feature space. This enables comparison of the data, which is difficult to do in the original high-dimensional space.

Yamanishi [24] outlined a distance metric learning method that can link heterogeneous objects such as compounds and proteins on the basis of two mappings of the heterogeneous objects to a common Euclidean space. We found in our experiments that this linear function works well for resolving entities in comparison to the other dimensionality reduction methods.

We thus used the conventional LPP method $[10 ; 24]$ to resolve entities. We first describe the use of consensus labels and then describe our proposed multi-Lap method with two weighting measures.

\subsection{LPP with Consensus Labels}

Linear projection $\mathbf{W}$ from an original $D$-dimensional feature space to a $d$-dimensional latent feature space is derived from training data consisting of data objects known to have or not to have links between them. If data objects $\mathbf{x}$ and $\mathbf{y}$ are assumed to have a known link, the distance between them in latent space should be as small as possible.

$$
\|\mathbf{W} \mathbf{x}-\mathbf{W y}\|
$$

The link between two data objects with unknown link status is predicted on the basis of the distance between them after they are mapped to the latent space by using $\mathbf{W}$.

Assume a set of $N$ training data objects $\mathbf{x}_{1}, \ldots, \mathbf{x}_{N}$ in $\mathbb{R}^{D}$. The process commonly used for finding the optimal projection matrix $\mathbf{W}^{*}$ that makes linked nodes close to each other is based on the method of He and Niyogi [10].

$$
\mathbf{W}^{*}=\underset{\mathbf{W}}{\arg \min } \sum_{i, j} \mathbf{A}_{i j}\left\|\mathbf{W} \mathbf{x}_{i}-\mathbf{W} \mathbf{x}_{j}\right\|_{2}^{2},
$$

where $\|\cdot\|_{2}$ is the Euclidean norm (2-norm), and $A_{i j}$ represents the link status between the data objects in the training data set, which 
can be defined as an adjacency matrix:

$$
A_{i j}= \begin{cases}1 & \text { if } \mathbf{x}_{i} \text { and } \mathbf{x}_{j} \text { have a link } \\ 0 & \text { otherwise. }\end{cases}
$$

We use consensus labels obtained by MV as the adjacency matrix. The problem can be rewritten as a generalized eigenvector problem:

$$
\begin{gathered}
\mathbf{W}^{*}=\underset{\mathbf{W}}{\arg \min } \operatorname{tr}\left(\mathbf{W} \boldsymbol{\Phi}^{\top} \mathbf{L} \boldsymbol{\Phi} \mathbf{W}^{\top}\right) \\
\text { s. t. } \mathbf{W} \boldsymbol{\Phi}^{\top} \mathbf{D} \boldsymbol{\Phi} \mathbf{W}^{\top}=\mathbf{I}_{d},
\end{gathered}
$$

where $\boldsymbol{\Phi}$ is the design matrix defined as $\boldsymbol{\Phi}=\left[\mathbf{x}_{1}, \ldots, \mathbf{x}_{N}\right]^{\top}, \mathbf{D}$ is a diagonal degree matrix in which the entries are column sums of $\mathbf{A}, \mathbf{D}_{i i}=\sum_{j} \mathbf{A}_{i j}$, and $\mathbf{L}$ is the Laplacian matrix defined by $\mathbf{L}=\mathbf{D}-\mathbf{A}$. $\mathbf{I}_{d}$ is the $d \times d$ identity matrix. We use the constraint to avoid the trivial solution $(\mathbf{W}=0)$ and ensure the uniqueness of the solution.

Solving this optimization problem is equivalent to solve the following generalized eigenvalue problem.

$$
\boldsymbol{\Phi} \mathbf{L} \boldsymbol{\Phi}^{\top} \mathbf{w}=\lambda \boldsymbol{\Phi} \mathbf{D} \boldsymbol{\Phi}^{\top} \mathbf{w} .
$$

By finding d eigenvectors with the smallest positive eigenvalues we can get the optimal linear projection matrix $\mathbf{W}^{*}$.

\subsection{Combination of Multiple Laplacians}

Though we can solve entities with aggregated crowdsourced labels, the information on the distribution of workers' judgment is missing in the learning process, e.g., the consensus label obtained by MV is truncated to 0 or 1 . We introduce a combination of multiple Laplacians to entity resolution to directly derive an accurate model from crowd-generated labels. The process of finding the optimal projection matrix can be seen as

$$
\mathbf{W}^{*}=\underset{\mathbf{W}}{\arg \min } \sum_{t}^{T} \sum_{i, j} \mathbf{A}_{i j}^{(t)}\left\|\mathbf{W} \mathbf{x}_{i}-\mathbf{W} \mathbf{x}_{j}\right\|_{2}^{2},
$$

where the adjacency matrix $\mathbf{A}^{(t)}$ is defined as $\mathbf{A}^{(t)}=\left\{\mathbf{A}_{i j}^{(t)}\right\}$, which is provided by worker $t . T$ is the number of crowd workers.

The problem can be also formulated as an optimization problem:

$$
\mathbf{W}^{*}=\underset{\mathbf{W}}{\arg \min } \operatorname{tr}\left(\mathbf{W} \boldsymbol{\Phi}^{\top} \sum_{t}^{T} \mathbf{L}^{t} \mathbf{\Phi} \mathbf{W}^{\top}\right),
$$

where $\mathbf{L}^{t}$ is the Laplacian matrix corresponding to adjacency matrix $\mathbf{A}^{(t)}$, which is defined the same as $\mathbf{A}$ in the LPP method, $\mathbf{A}_{i j}^{(t)}=1$ if $\mathbf{x}_{i}$ and $\mathbf{x}_{j}$ have a link otherwise $\mathbf{A}_{i j}^{(t)}=0$.

\subsubsection{Weighting Workers}

The model derived for a more qualified worker has a higher degree of accuracy. To compensate for the variability in the accuracy of the crowd workers and thereby get a more accurate true model, we impose an additional weighting measure on the learning process: we assign higher weights to more accurate workers.

As a result, the process of finding the optimal projection matrix can be rewritten as

$$
\mathbf{W}^{*}=\underset{\mathbf{W}}{\arg \min } \sum_{t}^{T} \sum_{i, j} r_{t} \mathbf{A}_{i j}^{(t)}\left\|\mathbf{W} \mathbf{x}_{i}-\mathbf{W} \mathbf{x}_{j}\right\|_{2}^{2},
$$

where $r_{t}$ is the weighting parameter.
The problem can also be formulated as an optimization problem:

$$
\mathbf{W}^{*}=\underset{\mathbf{W}}{\arg \min } \operatorname{tr}\left(\mathbf{W} \boldsymbol{\Phi}^{\top} \sum_{t}^{T} r_{t} \mathbf{L}^{t} \boldsymbol{\Phi} \mathbf{W}^{\top}\right) .
$$

This means that the problem can be reduced to a generalized eigenvalue problem:

$$
\boldsymbol{\Phi} \sum_{t}^{T} r_{t} \mathbf{L}^{t} \boldsymbol{\Phi}^{\top} \mathbf{w}=\lambda \boldsymbol{\Phi} \boldsymbol{\Phi}^{\top} \mathbf{w} .
$$

The optimal projection matrix $\mathbf{W}^{*}$ is obtained by finding the $d$ eigenvectors with the smallest positive eigenvalues. After the data objects are mapped to the $d$-dimensional space by using projection matrix $\mathbf{W}^{*}$, the links are identified on the basis of the distance between them, and the prediction accuracy can be calculated by comparing the predicted result with the gold standard.

To find the $d$ smallest eigenvectors can be problematic, so we found the eigenvectors for the largest positive eigenvalues of the generalized eigenvalue problem by setting $\lambda=1 \backslash \mu$. Then, the final generalized eigenvalue problem becomes:

$$
\boldsymbol{\Phi} \sum_{t}^{T} r_{t} \mathbf{L}^{t} \boldsymbol{\Phi}^{\top} \mathbf{w}=\mu \boldsymbol{\Phi} \boldsymbol{\Phi}^{\top} \mathbf{w} .
$$

Three weighting measures were used to represent crowd worker credibility. One values the weight of each worker as the degree of similariy, one uses mutual dependency principles to score the weight of each worker and without weighting. Without weighting, the labels provided by the crowd workers would be treated equally; that is, $r_{t}=1 / T$.

\section{Similarity.}

If we assume that most workers give true labels, a worker is more qualified if the labels provided by the worker are more similar to the labels provided by other workers, so a higher weight should be given to that worker. The similarity between labels $\mathbf{A}_{t_{i}}$ provided by worker $t_{i}$ and labels $\mathbf{A}_{t_{j}}$ provided by worker $t_{j}$ is defined as

$$
\operatorname{Sim}_{t_{i} t_{j}}=\frac{\left(\mathbf{A}_{t_{i}} \cdot \mathbf{A}_{t_{j}}\right)}{\left\|\mathbf { A } _ { t _ { i } } \left|\left\|\mid \mathbf{A}_{t_{j}}\right\|\right.\right.} .
$$

Thus, the weight of worker $t_{i}$ is defined as the sum of the similarities:

$$
r_{t_{i}}=\sum_{j \neq i, j \in \mathcal{T}} \operatorname{Sim}_{t_{i} t_{j}}
$$

where $\mathcal{T}$ is the set of crowd workers, $\mathcal{T}=\{1, \ldots, T\}$. In practice, we use normalization to make the sum of the weights of all workers equal to 1 .

\section{Mutual Dependence.}

A worker-labeled page pair can be modeled as a bipartite graph in which the label given by a worker to a pair of Web pages can be seen as a directed edge that carries the "opinion" of a source node towards a target node. From the perspective of the target node (a pair of Web pages), the edge can be identified as "agreeing"or "disagreeing"by determining whether the opinion carried by the edge agrees with the majority voting opinion for the target node. In our case, if most workers label a pair of Web pages $p$ as " 0 ," worker $t_{1}$ labels it as " 1 ," and worker $t_{2}$ labels it as " 0 ," edge $\left(t_{1}, p\right)$ is a disagreeing edge, and edge $\left(t_{2}, p\right)$ is an agreeing edge.

We use the mutual dependency principles proposed by Dai et al. [5] to evaluate of crowd worker credibility. These principles include the positive and negative mutual dependencies. An agreeing 
Table 1: Training data and test data (Web pages) for each person name.

\begin{tabular}{|l|c|c|}
\hline & Trainning data & Test data \\
\hline Abby Watkins & 60 & 52 \\
\hline Alvin Cooper & 40 & 47 \\
\hline Armando Valencia & 30 & 29 \\
\hline David Lodge & 40 & 50 \\
\hline Jerry Hobbs & 40 & 48 \\
\hline Michael Howard & 40 & 48 \\
\hline Patrick Killen & 40 & 48 \\
\hline Paul Clough & 30 & 34 \\
\hline Stephan Johnson & 40 & 35 \\
\hline Thomas Baker & 30 & 37 \\
\hline
\end{tabular}

edge has a positive mutual dependency on its source and target: a worker is more credible if he or she gives a label representing an agreeing edge to a pair of Web pages that are related to the same real world entity; similarly, a worker is less credible if he or she gives a label representing an agreeing edge to a pair of Web pages that are not related to the same real world entity. A disagreeing edge acts as a negative mutual dependency: a worker is less credible if he or she gives a label representing a disagreeing edge to a pair of Web pages that are related to a real world entity; a worker is more credible if he or she gives a label representing a disagreeing edge to a pair of Web pages that are not related to a real world entity.

Given bipartite graph $G=\langle\mathcal{T} \cup \mathcal{P}, E, S\rangle$, where $\mathcal{T}$ is the set of crowd workers, $\mathcal{P}=\left\{p_{1}, \ldots, p_{|\mathcal{P}|}\right\}$ is a set of Web page pairs, $E \subset \mathcal{T} \times \mathcal{P}$ is a set of directed edges derived from the labels given by the workers to pairs of pages, and $S=\left\{s_{i j}\right\}$ is the set of labels attached to the edges. If edge $\left(t_{i}, p_{j}\right)$ is an agreeing edge, $s_{i j}=0$; otherwise, $s_{i j}=1$.

Let $\mathfrak{t}_{\mathfrak{i}}$ be the weight assigned to worker $t_{i}$ and $\mathfrak{p}_{j}$ be the weight assigned to Web page pair $p_{j}$. The corresponding edge is $\left(t_{i}, p_{j}\right)$. The use of the mutual dependency principles means that, if $s_{i j}=0$, $\mathfrak{t}_{\mathfrak{i}}$ mirrors $\mathfrak{p}_{\mathfrak{j}}$, i.e., $\mathfrak{t}_{\mathfrak{i}}=\mathfrak{p}_{\mathfrak{j}}$; if $s_{i j}=1, \mathfrak{t}_{\mathfrak{i}}$ is the opposite of $\mathfrak{p}_{\mathfrak{j}}$, i.e., $\mathfrak{t}_{\mathfrak{i}}=1-\mathfrak{p}_{j}$. The edges are treated equally due to the absence of information on the relative credibility of the connected crowd workers.

$$
\left\{\begin{array}{l}
\mathfrak{t}_{\mathfrak{i}}=A V G_{p_{j}:\left(t_{i}, p_{j}\right) \in E}\left(1-2 s_{i j}\right) \mathfrak{p}_{j}+s_{i j} \\
\mathfrak{p}_{\mathfrak{j}}=A V G_{t_{i}:\left(t_{i}, p_{j}\right) \in E}\left(1-2 s_{i j}\right) \mathfrak{t}_{\mathfrak{i}}+s_{i j}
\end{array}\right.
$$

These formulae are converted into matrix form, and an iterative algorithm is used to compute the scores for the crowd workers. The score for each worker ranges from 0 to 1 . Scores in the range $[0,0.5)$ are considered workers in low credibility, and those in the range $[0.5,1)$ are considered high credibility.

\section{CROWDSOURCED LABELING FOR WEB ENTITY RESOLUTION}

In this section, we introduce the method we used for obtaining crowdsourced labels and the majority voting $(M V)$ method we used for aggregating the labels. Then, we evaluate the label quality of an actual crowdsourced data set.

\subsection{Data Set}

We used data obtained from the Searching Information about Entities in the Web (WEPS) dataset ${ }^{1}$, which provides Web pages

\footnotetext{
${ }^{1}$ http://nlp.uned.es/weps/weps-1/weps1-data
}

for given person names, especially for very common names that have high ambiguity on the Web and for names of famous or historical people. This dataset also provides a gold standard for Web pages containing each person's name, and these Web pages were well clustered by experts. The experts were asked to decide, on the basis of the contents, whether the person names on two Web pages referred to the same person in the real world. The number of Web pages used as training data and as test data for each person name are shown in Table 1 (blank pages were removed). We used tf-idf (the term frequency-inverse document frequency) as the feature.

\subsubsection{Crowd-generated Labels}

We used the Lancers crowdsourcing service ${ }^{2}$ to allocate HITs to crowd workers. Each HIT contained $n$ Web page URLs. The workers were instructed to open these pages one by one and find all the ones that referred to the same entity in the real world. A drop-down list on the HIT page enabled a worker to assign the same id number to the Web pages considered to refer to the same real world entity. Since we structured the HITs as the clustering of $N$ Web pages, we had to specify a sampling scheme. If we had structured the HITs as simply separate Web pages in disjoint sets, it would have been impossible to infer a clustering over the entire data set because we would not have known whether two Web pages in different HITs were in the same cluster or not. Therefore, the Web pages had to be members of multiple HITs.

We used a random sampling scheme [19; 9] in which a simple parameter $V$ is used to control the level of sampling redundancy. We set $V$ to be the number of HITs to which a Web page belonged. This meant that each Web page was voted on $V$ times by the crowd workers. We set $n$ to 10 or 20 depending on the number of Web pages available for the person name and $V$ to 5 . After removing the blank Web pages in WEPS, we had 30 Web pages as training data for person names "Armando Valencia," "Paul Clough," "Thomas Baker ( $n=10), 60$ for "Abby Watkins " and 40 for the other person names $(\mathrm{n}=20)$. The total number of data items was $N$. Thus, the total number of different HITs was

$$
H=N V / n \text {. }
$$

Because individual crowd workers often exhibit highly variable labeling accuracy, we asked multiple crowd workers to perform each HIT so that we could derive a single consensus label. Parameter $R$ is the number of crowd workers who performed the same HIT. Therefore, the total number of HITs generated was

$$
H=N V R / n \text {. }
$$

In the experiment described below, each HIT was assumed to be performed five times $(R=5)$.

\subsubsection{Consensus Labels}

Majority voting was used to generate consensus labels for the LPP method in two ways: to derive a single consensus label for each crowd worker and to generate the final consensus labels for all crowd workers. Since Web pages were assigned to multiple HITs, a worker could label a pair of Web pages several times. The two Web pages were assumed to be linked once the same id number was assigned to them by the same person.

As mentioned, simple MV was used to compute the final consensus labels (weighting each worker's vote equally). Consider a set of noisy labels $\left\{y_{i j}^{t}\right\}$ for data items pair $\left(\mathbf{x}_{i}, \mathbf{x}_{j}\right)$, where $y_{i j}^{t} \in\{0,1\}$ is a noisy label provided by the $t-$ th worker $(t \in\{1, \ldots, T\})$. The set of workers who labeled this pair is defined as $\mathcal{J}_{t} \subseteq\{1, \ldots, T\}$.

\footnotetext{
${ }^{2}$ http://www.lancers.jp
} 
Abby Watkins

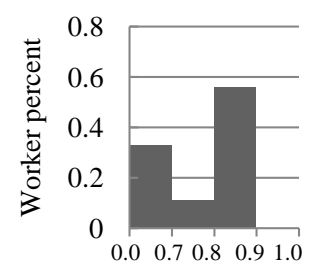

Michael Howard

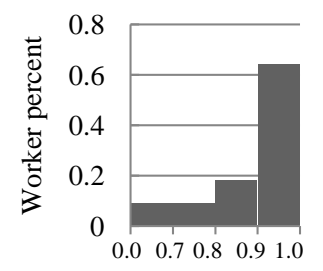

Alvin Cooper

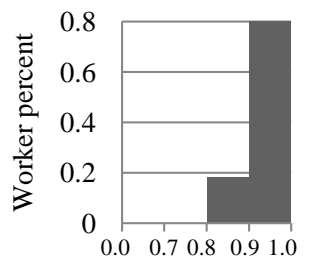

Patrick Killen

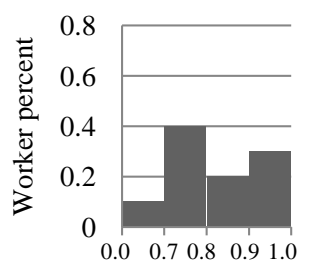

Armando Valencia

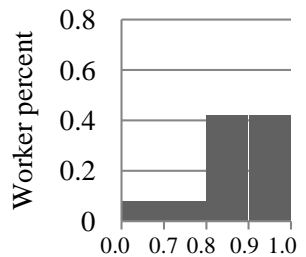

Paul Clough

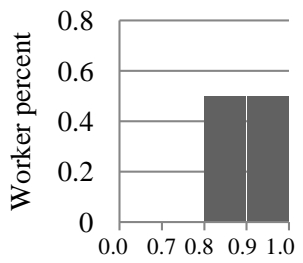

David Lodge

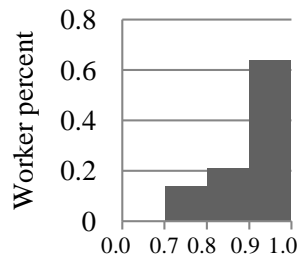

Stephan Johnson

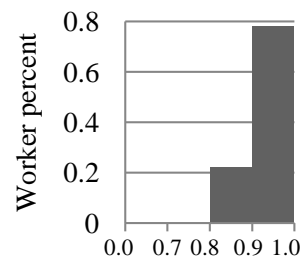

Jerry Hobbs

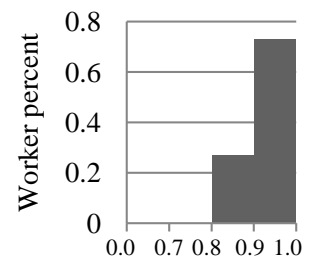

Thomas Baker

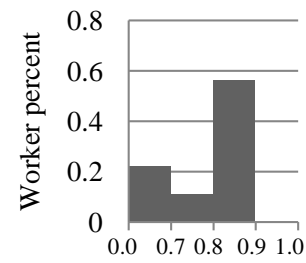

Figure 2: Accuracy of labels provided by individual workers.

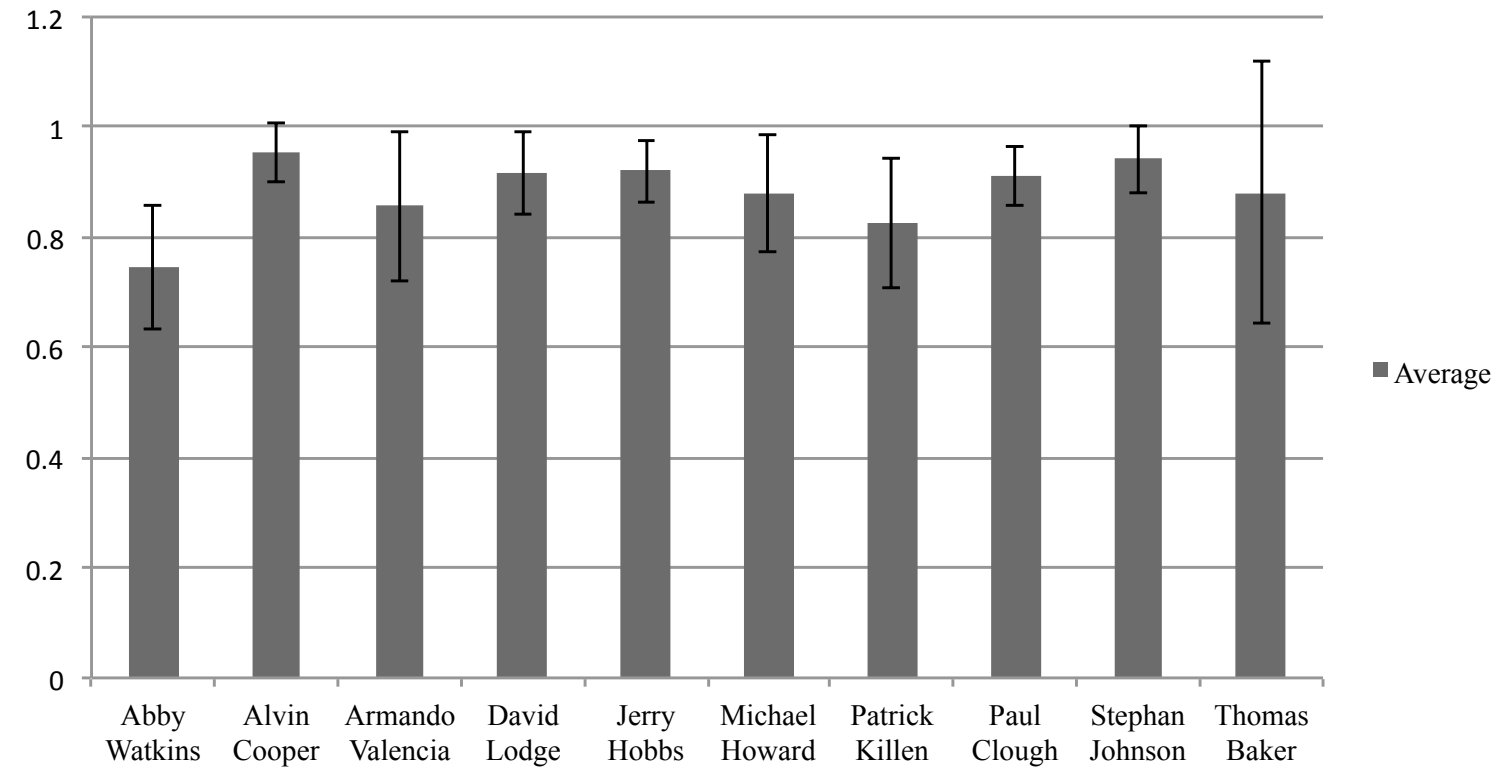

Figure 3: Average accuracy and standard deviation for each person name.

The consensus label $y_{i j}$ for this pair derived from majority voting is given by

$$
y_{i j}= \begin{cases}1 & \text { if } \sum_{t \in \mathcal{J}_{t}} y_{i j}^{t}>\left|\mathcal{J}_{t}\right| / 2 \\ 0 & \text { if } \sum_{t \in \mathcal{J}_{t}} y_{i j}^{t}<\left|\mathcal{J}_{t}\right| / 2 \\ \text { random } & \text { otherwise. }\end{cases}
$$

\subsection{Label Quality}

Using the methods described above, we collected actual crowdsourced data for ten person names ("Abby Watkins," "Alvin Cooper," "Armando Valencia," "David Lodge, ” "Jerry Hobbs," "Michael Howard,” "Patrick Killen,” "Paul Clough,” "Stephan Johnson,” "Thomas
Baker,") and generated consensus labels. We estimated the label quality of the crowdsourced data by comparing both the labels provided by individual workers and the consensus labels estimated by MV with the gold standard.

The experimental results presented in Figure 2 and Figure 3 and 4 show that the accuracy of the labels provided by individual workers was satisfactory although there was some dispersion of accuracy across workers (the average accuracy was above $80 \%$ except for Abby Watkins, and the standard deviation was mostly around $10 \%)$. The accuracy for the consensus labels was mostly around $80 \%$ and went as high as $90 \%$. Since entity resolution is difficult, the $80 \%$ accuracy is higher than predicted and indicates that it is 


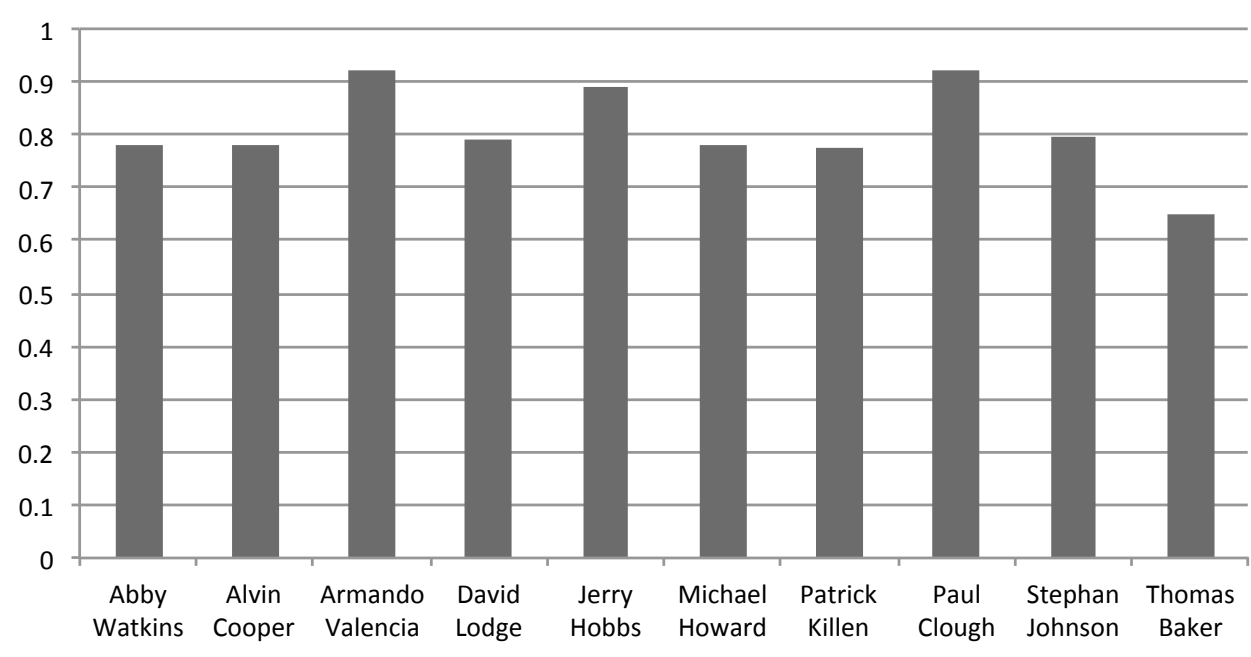

- Consenus labels

Figure 4: Accuracy of consensus labels.

possible to derive an accurate entity resolution model using these methods.

Here, we give only a general idea of how we can generate consensus labels by using crowdsourcing to resolve entities. Though there are some well-know techniques (e.g., expectation maximization) that may outperform MV, the labeling accuracy of MV is sufficient for our purposes here. The prediction accuracy of the LPP method with consensus labels was less than that of the gold standard no matter how much the labeling accuracy was improved, while experiment results given in section 4 show that the proposed multi-Lap method has accuracy comparable to or better than that of the LPP method with the gold standard.

\section{EVALUATION}

We experimentally evaluated the prediction accuracy of the LPP method using consensus labels and the multi-Lap method with three weighting measures by implementing it on the Matlab platform (Matlab R2011b) and using the built-in eigs function to solve the generalized eigenvalue problem. The dimension of the latent feature space was set to ten.

We estimated the prediction accuracy of the conventional LPP method [10] for the ground truth labels and for the consensus labels of the crowdsourced data. We also evaluated the prediction accuracy of the multi-Lap method without weighting (Uni), with weighting based on similarity (Sim), and with weighting based on mutual dependency (Mut). We used the AUC (area under the curve) values to measure the prediction.

Generally, for our proposed multi-Lap method, the accuracies using crowd-sourced data were comparable to or better than the conventional LPP method using gold standard and were superior to the LPP method using consensus labels eight out of ten cases in Figure 5.

However, interestingly, the multi-Lap method sometimes outperformed the LPP method using the gold standard, e.g., "Alvin Cooper" (Mut), "Armando Valencia " (Mut). In entity resolution, some Web pages are ambiguous even for human experts. Especially in certain difficult cases, the labels have to be decided to be a certain value that may cause overfitting. In the multi-Lap method, such difficult cases are treated without aggregating the labels.

By using the mutual dependency principles to calculate the cred- ibility of crowd workers, we can get the correct label even if the majority of the crowd workers give an incorrect label. The multiLap method with mutual dependency had better performance than the others two weighting measures five out of ten times and had performance comparable to the LPP method using the gold standard even for two exceptional cases "Patrick Killen" and "Paul Cloug".

Comparing the accuracy distributions in Figure 2 with the prediction accuracy, when most workers had virtually the same level of labeling skill ( "Jerry Hobbs", "Stephan Johnson"), the LPP method with consensus labels had higher prediction accuracy than the multi-Lap method. However, when most workers were in low labeling skill, as they did For "Abby Watkins", "Patrick Killen" and "Thomas Baker", the multi-Lap method still provided performance comparable to the LPP method using the gold standard.

Furthermore, from the results in Figure 6, we can see that our Multi-lap method always performs as least as fast as the LPP method with the true labels and the LPP method with the consensus labels. Though our Multi-lap with mutual dependency relatively the most time-consuming, most of the time is less than 0.1 second difference.

\section{CONCLUSION}

We have evaluated the label quality of actual crowdsourced data for entity resolution and compared the prediction accuracy of our proposed combination of multiple Laplacians (with and without weighting) with that of the conventional locality preserving projection method using consensus labels. Analysis of label quality showed that the accuracy of labels provided by individual workers was satisfactory while there was some dispersion of accuracy across workers. The prediction accuracy of the combination of multiple Laplacians method using crowd-sourced labels was comparable to or better than the LPP method using the gold standard and was superior to the LPP method using the consensus labels.

Our combination of multiple Laplacians method with mutual dependency had better performance than the other two weighting measures. However, the multiple Laplacians methods with mutual dependency cannot win in every case, a better weighting measurement is still in need. Our future work is solving the measure used for assigning weights to workers in the learning process. 


\begin{tabular}{|c|c|c|c|c|c|}
\hline & \multirow{2}{*}{$\begin{array}{c}\text { LPP } \\
\text { (True Label) }\end{array}$} & \multirow{2}{*}{$\begin{array}{c}\text { LPP } \\
\text { (Consensus Label) }\end{array}$} & \multicolumn{3}{|c|}{ Multi-LAP } \\
\hline & & & Uni & Mut & Sim \\
\hline Abby Watkins & 0.730 & 0.654 & 0.634 & 0.637 & 0.715 \\
\hline Alvin Cooper & 0.596 & 0.502 & 0.589 & 0.617 & 0.577 \\
\hline Armando Valencia & 0.532 & 0.556 & 0.517 & 0.569 & 0.533 \\
\hline David Lodge & 0.543 & 0.506 & 0.556 & 0.584 & 0.557 \\
\hline Jerry Hobbs & 0.523 & 0.560 & 0.508 & 0.508 & 0.503 \\
\hline Michael Howard & 0.545 & 0.549 & 0.564 & 0.532 & 0.509 \\
\hline Patrick Killen & 0.632 & 0.538 & 0.675 & 0.630 & 0.625 \\
\hline Paul Clough & 0.528 & 0.512 & 0.510 & 0.524 & 0.529 \\
\hline Stephan Johnson & 0.657 & 0.658 & 0.570 & 0.508 & 0.507 \\
\hline Thomas Baker & 0.636 & 0.533 & 0.509 & 0.691 & 0.501 \\
\hline
\end{tabular}

Figure 5: AUC values.

Abby Watkins

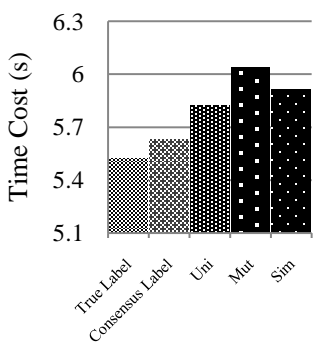

Michael Howard

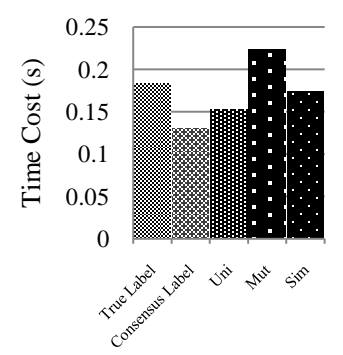

Alvin Cooper

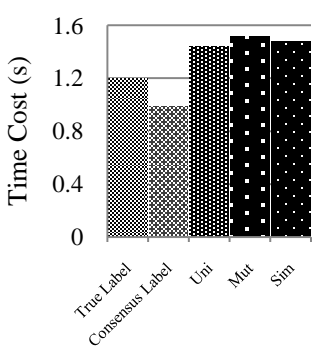

Patrick Killen

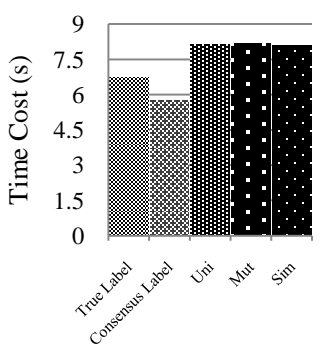

Armando Valencia

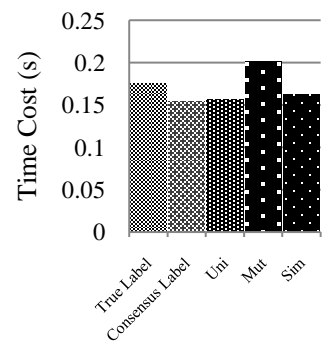

Paul Clough

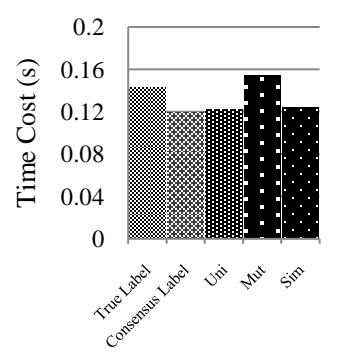

David Lodge

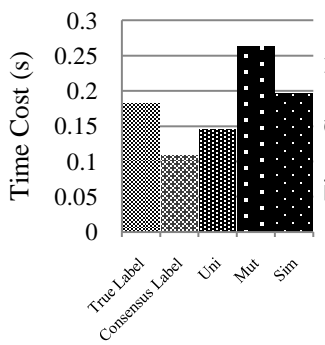

Stephan Johnson

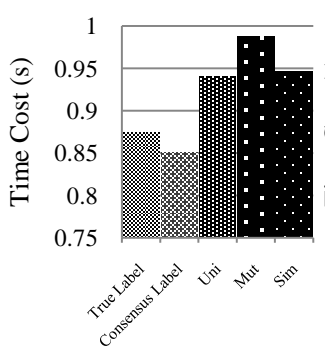

Jerry Hobbs

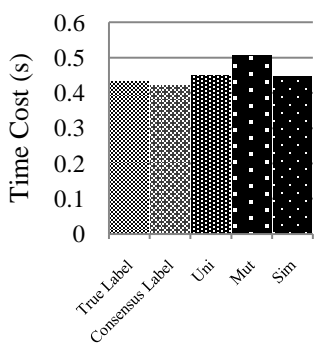

Thomas Baker

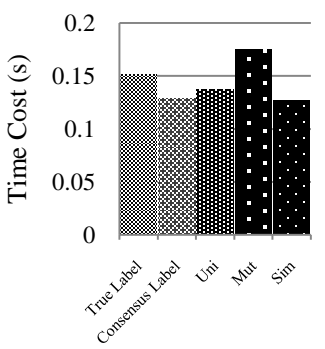

Figure 6: Time cost (CPU: Intel Core i7-3770 3.40GHz*2, memory: 8.00GB, operating system: Windows 7 Professional).

\section{REFERENCES}

[1] R. Al-Kamha and D. W. Embley. Grouping search-engine returned citations for person-name queries. In Proceedings of the 6th Annual ACM international Workshop on Web information and Data management, pages 96-103, 2004.

[2] R. Bekkerman and A. McCallum. Disambiguating Web appearances of people in a social network. In Proceedings of the 14th international conference on World Wide Web $(W W W$ ), pages 463-470, 2005.

[3] M. Bilenko, W. W. Cohen, S. Fienberg, R. J. Mooney, and P. Ravikumar. Adaptive name-matching in information integration. IEEE Intelligent Systems, 18(5):16-23, 2003.

[4] M. Bilenko and R. J. Mooney. Adaptive duplicate detection using learnable string similarity measures. In Proceedings of the 9th ACM SIGKDD International Conference on Knowledge Discovery and Data Mining (KDD), pages 39-48, 2003.

[5] H. Dai, F. Zhu, E. Lim, and H. Pang. Detecting anomalies in bipartite graphs with mutual dependency principles. 12th IEEE International Conference on Data Mining (ICDM), pages 171-180, 2012.

[6] A. P. Dawid and A. M. Skene. Maximum likelihood estimation of observer error-rates using the EM algorithm. Journal of the Royal Statistical Society. Series C, 1979.

[7] O. Dekel and O. Shamir. Vox populi: Collecting high-quality labels from a crowd. In Proceedings of the Conference on 
Learning Theory (COLT), 2009.

[8] W. S. Euijong, M. Julian, and G. M. Hector. Compare me maybe: Crowd entity resolution interfaces. In Technical Report, Standford InfoLab, 2012.

[9] R. Gomes, P. Welinder, A. Krause, and P. Perona. Crowdclustering. In Advances in Neural Information Processing 24 (NIPS), 2011.

[10] X. He and P. Niyogi. Locality preserving projections. In Advances in Neural Information Processing 16 (NIPS), 2004.

[11] H. Kajino, Y. Tsuboi, and H. Kashima. A convex formulation for learning from crowds. In Proceedings of the 26th AAAI Conference on Artificial Intelligence (AAAI), 2012.

[12] R. Kimura, S. Oyama, H. Toda, and K. Tanaka. Creating personal histories from the Web using namesake disambiguation and event extraction. In Proceedings of the 7th International Conference on Web Engineering (ICWE), pages 400-414, 2007.

[13] G. S. Mann and D. Yarowsky. Unsupervised personal name disambiguation. In Proceedings of the 7th Conference on Natural Language Learning at HLT-NAACL 2003, pages 33-40, 2003.

[14] A. Marcus, E. Wu, D. Karger, S. Madden, and R. Miller. Human-powered sorts and joins. In Proceedings of the VLDB Endowment, pages 13-24, 2011.

[15] A. G. Parameswaran, H. Garcia-Molina, H. Park, N. Polyzotis, A. Ramesh, and J. Widom. Crowdscreen: algorithms for filtering data with humans. In Proceedings of the 2012 ACM SIGMOD International Conference on Management of Data, pages 361-372, 2012.

[16] S. Sarawagi and A. Bhamidipaty. Interactive deduplication using active learning. In Proceedings of the 8th ACM SIGKDD Conference on Knowledge Discovery and Data Mining (KDD), pages 269-278, 2002.

[17] V. S. Sheng, F. Provost, and P. G. Ipeirotis. Get another label? improving data quality and data mining using multiple, noisy labelers. In Proceedings of the 14th ACM SIGKDD Conference on Knowledge Discovery and Data Mining (KDD), pages 614-622, 2008.

[18] R. Snow, B. O'Connor, D. Jurafsky, and A. Y. Ng. Cheap and fast-but is it good?: evaluating non-expert annotations for natural language tasks. In Proceedings of the Conference on Empirical Methods in Natural Language Processing (EMNLP), pages 254-263, 2008.

[19] A. Strehl and J. Ghosh. Cluster ensembles - a knowledge reuse framework for combining multiple partitions. Journal of Machine Learning Research, 3:583-617, 2003.

[20] S. Tejada, C. A. Knoblock, and S. Minton. Learning domain-independent string transformation weights for high accuracy object identification. In Proceedings of the 8th ACM SIGKDD Conference on Knowledge Discovery and Data Mining (KDD), pages 350-359, 2002.

[21] X. Wan, J. Gao, M. Li, and B. Ding. Person resolution in person search results: Webhawk. In Proceedings of the 14th ACM International Conference on Information and Knowledge Management (CIKM), pages 163-170, 2005.

[22] J. Wang, T. Kraska, M. J. Franklin, and J. Feng. CrowdER: crowdsourcing entity resolution. In Proceedings of the VLDB Endowment, pages 1483-1494, 2012.

[23] F. L. Wauthier and M. I. Jordan. Bayesian bias mitigation for crowdsourcing. In Advances in Neural Information Processing 24 (NIPS), pages 1800-1808, 2011.

[24] Y. Yamanishi. Supervised bipartite graph inference. In Advances in Neural Information Processing Systems 21 (NIPS), pages 1841-1848, 2009.

[25] H. Yang, A. Mityaginr, K. M. Svore, and S. Markov. Collecting high quality overlapping labels at low cost. In Proceedings of the 33rd International ACM SIGIR Conference on Research and Development in Information Retrieval (SIGIR), pages 459-466, 2010. 